\title{
Bodyguards under Cover: the Status of Individual Concepts
}

\author{
Magdalena Schwager \\ Frankfurt University
}

\section{Introduction}

Certain nouns like temperature and mayor seem to be systematically ambiguous between a function and a value reading. On the one hand, they can refer to a function that tells us for each moment of time what the temperature of a particular place is or who the mayor of a particular city is. On the other hand, they can refer to an individual which constitutes the actual value of the aforementioned function. The temperature paradox (attributed to Barbara Partee) evidences this difference: The subject position of an intensional verb like rise requires reference not only to the actual index, but also a temporal interval around it; that is, it appeals to the function. The second sentence specifies the actual value, to which properties of the function need not apply. (1) thus constitutes an invalid inference. ${ }^{1}$

(1) The temperature is rising.

The temperature is ninety.

Ninety is rising.

Löbner (1981) gives somewhat more natural examples of such fallacies with temperatures (cf. 2) and mayors (cf. 3). ${ }^{2}$

(2) Right now, the temperature of the air in my refrigerator is the same as the temperature of the air in your refrigerator.

The temperature of the air in my refrigerator is rising.

The temperature of the air in your refrigerator is rising.

(3) The mayor of Frankfurt is Petra Roth.

The mayor could have changed last January.

Petra Roth could have changed last January.

How should we account for this dual role of referring to functions or their values?

For many helpful comments I would like to thank the audiences of SALT XVI as well as of Semantiknetzwerk 6, DIP Amsterdam, GK Stuttgart, and Semantikzirkel Frankfurt.

${ }^{1}$ ninety is taken to abbreviate ninety degrees Fahrenheit and to refer to an abstract degree individual which can also be picked out by 32 degrees Celsius.

${ }^{2}$ Note that the last sentence of (3) also has a reading of partial change under which the individual picked out by the proper name Petra Roth changes with respect to a particular property, cf. Löbner (1979). In this paper, I focus exclusively on the reading where different individuals are picked out before and after the change. 


\section{Individual Concepts from the Lexicon}

\subsection{The Montagovian Solution}

The classical solution to the temperature paradox is given in Montague (1974) (PTQ). He assumes that, at an index, common nouns denote not just sets of individuals, $\langle e, t\rangle$, but rather sets of individual concepts, that is, of functions from indices to individuals. ${ }^{3}$ Common nouns thus have $\langle\langle s, e\rangle, t\rangle$-denotations. The model contains an abstract degree individual picked out by the type $e$-constant $90 \mathrm{~F}$, ninety (degrees Fahrenheit) is translated as $\lambda \boldsymbol{\lambda} . P_{(w, t)}(\lambda(w, t) .90 \mathrm{~F})$, the translates as Russellian quantifier, be as extensional identity:

$$
\begin{aligned}
& \text { the } \equiv \lambda P_{\langle\langle s, e\rangle, t\rangle} \lambda Q_{\langle\langle s, e\rangle, t\rangle} \cdot \exists x[\forall y[P(y) \leftrightarrow y=x] \wedge Q(x)] \\
& \text { be } \equiv \boldsymbol{\lambda} \mathscr{P}_{\langle s,\langle s,\langle\langle s, e\rangle, t\rangle\rangle\rangle} \boldsymbol{\lambda} \boldsymbol{x}_{\langle s, e\rangle} \cdot \mathscr{P}_{(w, t)}(\boldsymbol{\lambda}(\boldsymbol{w}, t) \boldsymbol{\lambda} \boldsymbol{y} \cdot \boldsymbol{x}(\boldsymbol{w}, t)=\boldsymbol{y}(\boldsymbol{w}, t))
\end{aligned}
$$

The PTQ-translation of the classical temperature paradox thus looks like (6):

$$
\begin{aligned}
& \text { a. } \exists x\left[\forall y\left[\operatorname{temperature}_{(w, t)}(y) \leftrightarrow x=y\right] \wedge \operatorname{rise}_{(w, t)}(x)\right] \\
& \text { b. } \exists x\left[\forall y\left[\operatorname{temperature}_{(w, t)}(y) \leftrightarrow x=y\right] \wedge x(w, t)=90 \mathrm{~F}\right] \\
& \text { c. } \operatorname{rise}_{(w, t)}(\lambda(w, t) .90 \mathrm{~F})
\end{aligned}
$$

It is easy to see that rising can be a property of the unique individual concept describing the salient temperature, but not of the individual picked out by the constant $90 \mathrm{~F}$ or the constant individual concept $\lambda(\boldsymbol{w}, t) . \mathbf{9 0 F}$. Thus, a solution to the temperature paradox is obtained.

\subsection{A Problem for the Montagovian Solution}

Dowty et al. (1981) discuss in some detail a problem for the Montagovian solution pointed out by Anil Gupta. ${ }^{4}$

(7) Necessarily, the temperature of the air in my refrigerator is always the same as the temperature of the air in your refrigerator.

The temperature of the air in my refrigerator is rising.

The temperature of the air in your refrigerator is rising.

Intuitively, (7) is valid. However, given the translations obtained from the PTQframework (cf. 8) and the interpretation of rise (cf. 9) it is predicted to be invalid.

\footnotetext{
${ }^{3}$ Throughout this paper, I translate LFs to a variant of Ty2: $e$ the type of individuals, $\boldsymbol{t}$ the type of truth values, $s$ the type of indices, which consist of a world and a time component. Interpretation [ $\cdot]$ proceeds with respect to a standard model $M$ and an assignment $g$, reference to which is mostly ignored for simplicity. Section 4.1 adds a further parameter $F$.

${ }^{4}(7)$ is a recast from Löbner (1981). Following Romero (2006), I insert always to ensure the habitual reading crucial for validity of the inference. She shows that syntactic decomposition into quantification over worlds and times already avoids many mispredictions; yet, certain syntactic assumptions or a meaning postulate are needed in addition.
} 
(8)
a. $\forall(\boldsymbol{w}, t) \exists \boldsymbol{x}\left[\forall \boldsymbol{y}\left[\right.\right.$ temperature-in-my-refrigerator $\left._{(\boldsymbol{w}, t)}(\boldsymbol{y}) \leftrightarrow \boldsymbol{x}=\boldsymbol{y}\right] \wedge$ $\exists z\left[\forall y\left[\operatorname{temperature-in-your-refrigerator}_{(w, t)}(y) \leftrightarrow z=y\right] \wedge\right.$ $x(w, t)=z(w, t)]]$
b. $\exists x\left[\forall y\left[\operatorname{temperature-in-my-refrigerator~}_{(w, t)}(y) \leftrightarrow x=y\right] \wedge \operatorname{rise}_{(w, t)}(x)\right]$
c. $\exists x\left[\forall y\left[\right.\right.$ temperature-in-your-refrigerator $\left.\left._{(w, t)}(y) \leftrightarrow x=y\right] \wedge \operatorname{rise}_{(w, t)}(x)\right]$

(9) $\llbracket$ rise $\rrbracket(w, t)\left(f_{\langle s, e\rangle}\right)=1$ iff there is an interval $T$ that includes $t$ and for all $t^{\prime}, t^{\prime \prime}$ in $T$ : if $t^{\prime}<t^{\prime \prime}$, then $f\left(w, t^{\prime}\right)<f\left(w, t^{\prime \prime}\right)$.

To see the failure, we consider a model $M$ with one world $w$, three temporal instants $t_{1}, t_{2}, t_{3}$ and the interpretation of the constants corresponding to Figure 2.2.

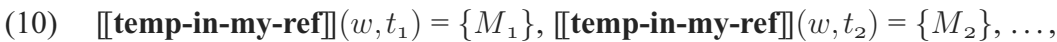
【temp-in-your-ref $\rrbracket\left(w, t_{1}\right)=\left\{Y_{3}\right\}$

$$
\begin{aligned}
& M_{1}=\left\{\left\langle\left(w, t_{1}\right), 15\right\rangle,\left\langle\left(w, t_{2}\right), 12\right\rangle,\left\langle\left(w, t_{3}\right), 8\right\rangle\right\}, \\
& M_{2}=\left\{\left\langle\left(w, t_{1}\right), 12.5\right\rangle,\left\langle\left(w, t_{2}\right), 25\right\rangle,\left\langle\left(w, t_{3}\right), 30\right\rangle\right\}, \ldots, \\
& Y_{3}=\left\{\left\langle\left(w, t_{1}\right), 5\right\rangle,\left\langle\left(w, t_{2}\right), 10\right\rangle,\left\langle\left(w, t_{3}\right), 20\right\rangle\right\}
\end{aligned}
$$

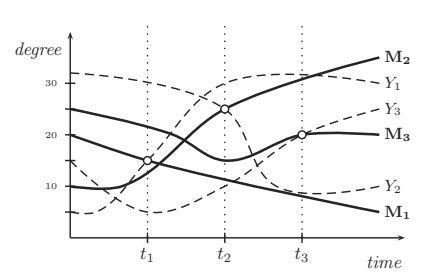

Figure 1: Graphical representation of the counter-model $M$.

(8a) is true because at each index the two functions picked out there as describing the temperatures in your and my refrigerator respectively assign the same value to that index. Moreover, at $t_{2}$, the function selected as describing the temperature in my refrigerator $\left(M_{2}\right)$ is rising (so, $8 \mathrm{~b}$ is true). Nevertheless, the one that in $t_{2}$ describes the temperature of your refrigerator $\left(Y_{2}\right)$ is falling (so, 8c is false).

At this point, the problem with the Montagovian solution is quite obvious: by employing constants of type $\langle s,\langle\langle s, e\rangle, t\rangle\rangle$, we have doubled the index dependence of temperature: At a fixed index, it denotes a set of functions that assign (degree-)individuals to indices (individual concepts); I call this the inner index dependence (IID). At the same time, it can denote different such sets at different indices; I call this the outer index dependence (OID).

To get around this surplus of variation that is responsible for the failure of intuitively valid inferences like (7), various strategies are conceivable: (i) a meaning postulate (= give up OID), (ii) intensions of Fregean definite descriptions (Lasersohn 2005) (= give up IID), (iii) conceptual covers (OID = semantics, IID = pragmatics), (iv) intensional identity (a stronger version of be), or (v) type shifts from 
index dependent sets of individuals/pairs to index independent sets of individual concepts (explored briefly in Schwager 2006; in detail in Nathan 2005).

The easiest solution (and most likely the one Montague himself would have gone for), is to employ a Meaning Postulate along the lines of (12). This ensures triviality of the outer index dependence and thus validity of (7). ${ }^{5}$

$$
\forall x \forall(w, t)\left[\alpha_{(w, t)}(x) \rightarrow \forall(w, t)\left[\alpha_{(w, t)}(x)\right]\right], \text { for } \alpha=\text { temperature, price, } . .
$$

Romero (2006) shows that under a more careful treatment of quantification over worlds and times and with attention to the difference between habitual and accidental identity even a weaker meaning postulate against variation over time within one world is sufficient. Still, as long as we need meaning postulates to avoid mispredictions w.r.t. functions and their values, I think we should worry about whether our analysis misses some deeper connection between them. ${ }^{6}$ Moreover, in Section 3.4, I will discuss a problem that does not find a straightforward-solution in terms of individual concepts coming from the lexicon. In this paper, I focus exclusively on the alternatives (ii) and (iii). I ignore the possibility to render the copula ambiguous (it generates too many inaccurate readings). In contrast, the alternative in terms of type-shifts as proposed by Nathan (2005) deserves careful comparison that is beyond the scope of this paper.

\section{Deriving Individual Concepts in the Course of the Derivation}

\subsection{Lasersohn: Intensions of Fregean Definite Descriptions}

According to Lasersohn (2005), outer index dependence is just the natural dependence of common nouns on the index of evaluation, and should thus be kept. But he considers inner index dependence to be forced by Montague's Russellian treatment of the (repeated in 13). Since some predicates (e.g. rise) require an argument of type $\langle s, e\rangle$, the restrictor has to be of the same type. Hence, at an index, temperature gets interpreted as a set of individual concepts instead of individuals.

$$
\text { the } \equiv \lambda P_{\langle\langle s, e\rangle, t\rangle} \lambda Q_{\langle\langle s, e\rangle, t\rangle} \cdot \exists x[\forall y[P(y) \leftrightarrow y=x] \wedge Q(x)]
$$

\footnotetext{
${ }^{5}$ Note that Lasersohn's (2005) version sketched in (12) fails to take into account the implicit argument. Therefore, (5) is still not predicted to be valid:

(i) At all worlds and times, the temperature of Cécile's refrigerator not of my refrigerator, is the same as the temperature of Ede's refrigerator.

The temperature of the Cécile's refrigerator is rising. intuitively $\Rightarrow ;$ Montague $+\mathrm{MP}(12)$ predict $\nRightarrow$

The temperature of Ede's refrigerator is rising.
}

Assume that at all indices, the individual concept $c$ records the temperature of Cécile's refrigerator, and apart from $(w, t)$, the temperature of Ede's refrigerator is recorded by $x_{e d e}$, and the one of mine by $x_{m}$ (so both are still temperatures). At $(w, t), x_{m}$ and $x_{e d e}$ are exchanged, but $x_{m}(w, t)=c(w, t)$. Even if the premises and (12) are true, the consequence need not be.

${ }^{6} \mathrm{Cf}$. Zimmermann (1999) for more general discussion of the status of meaning postulates. 
Lasersohn then argues that a Fregean treatment of definite descriptions (cf. 14) allows for an interesting alternative to capture the function/value ambiguity: let temperature denote a set of individuals at each index, that is, temperature starts out as 'actual temperature value'. Zooming in on a salient location/object, this is a singleton set. Therefore, uniqueness and existence presupposition of $\iota$ are met and the definite description the temperature is felicitous. To talk about the development of the salient object's temperature throughout time, we use the intension of the Fregean definite description. This is exactly the function that assigns each index the unique temperature value at that index, hence, an individual concept.

$$
\begin{aligned}
& \text { the } \equiv \lambda P_{\langle\mathrm{s},\langle\mathrm{e}, \mathrm{t}\rangle\rangle} \lambda Q_{\langle\langle s, e\rangle, t\rangle} \cdot Q\left(\lambda(\boldsymbol{w}, t) \cdot \iota u\left[P_{(w, t)}(u)\right]\right) \\
& \llbracket \iota u \phi \rrbracket^{g} \text { is the unique object } d \text { such that } \llbracket \phi \rrbracket^{g[\mathrm{u} / d]}=1 \\
& \text { if such an object } d \text { exists; undefined otherwise. }
\end{aligned}
$$

At all indices, $\lambda(\boldsymbol{w}, t) \cdot \iota \boldsymbol{u}\left[\right.$ temperature $\left._{(\boldsymbol{w}, t)}(\boldsymbol{u})\right]$ denotes the same function that picks out the temperature at each index. With temperature as a constant of type $\langle s,\langle e, t\rangle\rangle$, and rise untouched (type $\langle\langle s, e\rangle, t\rangle$ ), we obtain the following translations of the temperature-paradox and the my/your refrigerator puzzle:

$$
\begin{aligned}
& \text { a. } \operatorname{rise}_{(w, t)}\left(\lambda(w, t) \cdot \iota u\left[\operatorname{temperature}_{(w, t)}(u)\right]\right) \\
& \text { b. } \iota u\left[\operatorname{temperature}_{(w, t)}(u)\right]=\mathbf{9 0 F} \\
& \text { c. } \operatorname{rise}_{(w, t)}(\lambda(w, t) .90 \mathrm{~F})
\end{aligned}
$$

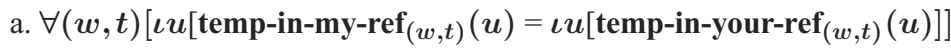

$$
\begin{aligned}
& \text { b. } \operatorname{rise}(\lambda(w, t) . \iota u[\operatorname{temp-in-my-ref}(w, t)(u)]) \\
& \left.\left.{\text { c. } \operatorname{rise}_{(w, t)}(\lambda(w, t) . \iota u[\text { temp-in-your-ref }}_{(w, t)}(u)\right]\right)
\end{aligned}
$$

Eventually, the translations of both temperature-paradox and temperature-price puzzle look satisfactory. The unintuitive multiplicity of temperature functions in the world has been removed. In addition, the types have been simplified considerably.

\subsection{When Uniqueness Fails}

Nevertheless, Lasersohn's solution faces a big problem. temperature is an inherently functional concept, hence, temperature as temperature at the salient location, will always denote a singleton set and thus never appear as the restrictor of a generalized quantifier (other than Russellian the). But if the temperatures of different locations/objects are under consideration as e.g. in the context of a weather station that monitors different citites, examples like (18) are fully natural. Romero (2006) considers this decisive to abandon Lasersohn's idea in favour of improving Montague's solution in terms of $\langle\langle s, e\rangle, t\rangle$-extensions, backing it up with a careful distinction between accidental and habitual identity as well as a meaning postulate.

(18) Every temperature is rising.

Since Lasersohn blames the uniform type assignment to restrictor and nuclear scope as the origin of inner index dependence, one could try to avoid it for quantifiers like every. But neither (19a) nor (19b) are viable. 

a. $\lambda P_{\langle\underline{\langle e, t\rangle}} \lambda Q_{\langle\langle s, e\rangle, t\rangle} \forall u_{\underline{\mathrm{e}}}[\boldsymbol{P}(\boldsymbol{u}) \rightarrow Q(\lambda(w, t) . u)]$
b. $\lambda \boldsymbol{P}_{\underline{\langle e, t\rangle}} \lambda Q_{\langle\langle s, e\rangle, t\rangle} \forall \boldsymbol{x}_{\underline{\langle s, e\rangle}}[\boldsymbol{P}(\boldsymbol{x}(\boldsymbol{w}, t)) \rightarrow Q(x)]$

Quantification over actual temperature values and predicating rise of their intension as in (19a) fails because $\lambda(w, t) . u$ is constant for any $u$ of type $e$. (19b) quantifies over all individual concepts whose value is (also) a temperature at the given index. This does not give the desired solution either. If the abstract degree individual $90 F$ occurs as a temperature at an index $(w, t)$, any function $f$ with $f(w, t)=90 F$ would have to be rising. But one can always find an individual concept to falsify this. ${ }^{7}$ However, I do not want to give up too quickly on Lasersohn's intuition that one can somehow extract functions from actual values. To extend his solution to examples like (18), the first step is to take seriously the relational (ultimately, functional) character of temperature: temperature really means temperature of an object/at a location. In 3.3, I investigate a way to make use of this in the course of the semantic derivation, in 3.4 I show two cases where it fails. I will then take a second step and argue that the individual concepts are not derived in the course of the semantic derivation, but are part of how we individuate individuals. That is, they come in at a pragmatic level only. This retains Lasersohn's insight that values are primary and functions are derived, but it deviates from his solution in deriving the functions at a different level.

\subsection{Extending Lasersohn by Quantifying over Implict Arguments in Semantics}

If we take into account that temperature is really a functional noun, we obtain a natural interpretation for (18) (repeated as 20) by quantifying over the implicit argument:

(20) Every temperature is rising. every object $x$ is such that the temperature of $x$ is rising

Generally, quantifiers seem to be able to bind implicit arguments (cf. Partee 1989). Evidence comes from examples like (21):

(21) Every farmer knows a neighbour. (preferred reading: Every farmer knows a neighbour of his.)

To extend Lasersohn's solution to examples like (18), I translate temperature as temperature $_{(w, t)}$ of type $\langle e,\langle e, t\rangle\rangle$. Before it combines with the definite article, its first argument has to be saturated (e.g. by a free variable present in the syntax). The quantificational determiners every, most,... are taken to be ambiguous; when they are to combine with a relational noun the first argument of which has not been saturated, the versions in (22) are used.

\footnotetext{
${ }^{7}$ E.g. assume $90 F$ is the temperature value of Frankfurt at $(w, t)$. Then, another individual concept $f^{t}{ }_{m}$ with the same value is the mirror temperature at $t$ of Frankfurt defined as in (i).

(i) for any world $w: f^{t}{ }_{m}(w, t)=f(w, t)$, and any amount of time $n, f^{t}{ }_{m}(w, t+n)=f(w, t-n)$ Now, at any index either the temperature in Frankfurt $f$ is falling, or $f^{t}{ }_{m}$ is falling, or both are constant.
} 
(22)

$$
\begin{aligned}
& \text { every }_{2} \text { translates into } \\
& \lambda P_{\langle s,\langle e,\langle e, t\rangle\rangle\rangle} \lambda Q_{\langle\langle s, e\rangle, t\rangle} \cdot \forall u\left[C(u) \rightarrow Q\left(\lambda(w, t) . \iota v\left[P_{(w, t)}(u)(v)\right]\right)\right] \\
& \text { most }_{2} \text { translates into } \\
& \lambda P_{\langle s,\langle e,\langle e, t\rangle\rangle\rangle} \lambda Q_{\langle\langle s, e\rangle, t\rangle \cdot \operatorname{most}(\lambda u \cdot C(u))\left(\lambda u \cdot Q\left(\lambda(w, t) \cdot \iota v\left[P_{(w, t)}(u)(v)\right]\right)\right)}
\end{aligned}
$$

E.g. (23a) is now translated as (23b). It is interpreted to be true iff for most contextually relevant objects $u$ it is the case that the function constituted by the intension of the unique temperature of $u$ is rising.

$$
\begin{aligned}
& \text { a. Most temperatures are rising. } \\
& \text { b. } \operatorname{most}(\lambda u . C(u))\left(\lambda u \cdot \operatorname{rise}_{(w, t)}\left(\lambda(w, t) . \iota v\left[\operatorname{temperature}_{(w, t)}(u)(v)\right]\right)\right)
\end{aligned}
$$

When quantifying over implicit arguments we should be on guard for the proportion problem, though. ${ }^{8}$ Consider (24a). Given that mother need not correspond to an injective function (two children can have the same mother), it is crucial that, rather than counting children, we count people who are mother of someone. ${ }^{9}$

a. Most mothers love their children.

b. Most children's mothers love them.

Now, temperature is clearly not injective on the value reading, but things are less clear on the function reading. In principle, it does not seem to be impossible that two objects have the same temperature at all worlds and times. Nevertheless, it is hard to tell if that should count as involving one or two temperatures for sentences like (23a). The analysis developed in this subsection amounts to counting two necessarily identical temperatures twice. Although intuitions are very weak, this seems to be quite acurate for (23a). Nevertheless, in the case of two cities sharing the same person as a mayor at all worlds and times, intuitions are rather that (on the function reading) this should count as one mayor only.

As it stands, the ambiguity in quantificational determiners overgenerates quite a bit: not only do we make the wrong predictions for non-classical quantification over non-injective functional nouns like mother, we also have to ban application to properly relational nouns. For (25a), more than one senator per state does not lead to a presupposition failure (and again, we do not want to count states). The desired reading is obtained by first turning the relational noun into a sortal one by anaphoric interpretation of the implicit argument or by existential closure (as in

\footnotetext{
${ }^{8}$ Possessive constructions as discussed by Barker (1995) differ in that respect:

(i) Most students' papers are too long.

For most students $x$ : $x$ 's papers are too long.

Thanks to the maximalization inherent in the possessive construction, quantification over extracted possessors (inverse linking) gives rise to the correct reading.

${ }^{9}$ The issue does not arise in classical quantification like (ia) which Löbner (1985) suggests to analyze as (ib):

(i) a. Every mother loves her child.

b. Every child's mother loves that child.
} 
$25 b)$. We can then apply ordinary quantification over individuals that are senators of some state.
a. Every senator was late.
b. Every $x$ such that there is a state $y$ and $x$ is senator from $y$ was late.

So, we are not absolutely sure if we would always count the right objects (intensionally equivalent temperatures), and there is still some overgeneration to be banned by restricting the solution to a proper sub-class of functional nouns. ${ }^{10}$ Still, implicit quantification carries some promise for nouns like temperature. In the following section I will discuss two cases that the approach fails to extend to.

\subsection{Two Types of Properly Relational Nouns}

The real problem for an extension of the Lasersohn-style solution comes from the fact that not all nouns in subject position of rise are functional. Hence, even if we quantify over the implicit argument, the individual concept obtained as the intension corresponding to the definite counterpart of the common noun phrase hidden in the syntactic subject is always undefined.

On the one hand, we find nouns that seem to describe sets of functions (and their respective values) at a given index. Löbner (1979) dubs them funktionenbündel (bundle of functions).
a. One value (of patient Smith) is rising.
b. Two of his critical values are rising.

Here, we do not want to count patients, but for a given patient (Smith) the number of functions that count as his critical values at that index and are rising. For the sentences to be true there need not be (and, for $26 \mathrm{~b}$ there certainly cannot be) a unique critical value of Smith. Intuitively, one would want to somehow stick in the different roles various critical values have with respect to Smith (one being his (unique) blood pressure, one his (unique) concentration of cholesterol,...). If this could be smuggled into the semantics, something like (27) might result. While meeting our intuitions for (26b), it is of course a mere stipulation.

$$
\begin{aligned}
& \mid\left\{f \in \left\{\lambda(w, t) . \iota u\left[\text { critical-value }(\text { smith })_{(w, t)}(u) \wedge P(\text { smith })(u) \mid\right.\right.\right. \\
& \left.P \in\{\text { bl-press, conc-chol, temperature }\}\} \mid \operatorname{rise}_{(w, t)}(f)\right\} \mid=3
\end{aligned}
$$

A second problem is constituted by what I call sets without roles.

(28) Three bodyguards (of Arnold) have changed.

As we are talking about the bodyguards of a particular person (namely Arnold), we cannot distinguish bodyguards by whose unique bodyguard each of them is. Thus, bodyguards are similar to critical values and different from temperatures. But we cannot rely on there being identifying roles that they play with respect to the person

\footnotetext{
${ }^{10}$ Maybe those that Nathan (2005) treats as functions to degrees on scales instead of to individuals.
} 
they are bodyguard of, either. In that, bodyguards are unlike critical values and cannot be treated along the lines of (27).

Nathan (2005) (cf. also Romero 2006) observes an interesting effect on the interpretation of change that I will refer to as Nathan's Puzzle. In my terminology, it relates to the distinction between functional nouns and funktionenbündel on the one hand, and sets without roles on the other. For (29a) to be true, the overall set of pictures on Jordan's wall has to change (set change SC), whereas for (29b), pointwise change (within the set; PC) is sufficient (that is, the overall set of governors may stay the same, but they swap jobs among themselves). ${ }^{11}$

a. The pictures on Jordan's wall have changed.

b. Three governors have changed.

To sum up, my extension of Lasersohn's solution within semantics proper fails to account for two sorts of relational nouns in subject position (namely, funktionenbündel and sets without roles). Moreover, we lack a solution to the interpretive behaviour of change as constituted by Nathan's puzzle. The original Montagovian solution (backed up with a meaning postulate against outer index dependence), fares well on the first part of the problem but fails to account for Nathan's puzzle. ${ }^{12}$

\section{Individual Concepts as Elements of Conceptual Covers}

\subsection{Interrogation and Quantification under Cover}

Neither individual concepts from the lexicon nor individual concepts derived in the course of the semantic derivation account for all the phenomena observed with nouns in subject positions of intensional verbs. Therefore, I would like to go back to the original motivation of individual concepts as identifying the individuals one wants to talk about. A formal account of the idea that belief attribution, as well as questioning and quantification proceed with respect to mehods of identification, is provided in Aloni (2000). ${ }^{13}$ Although, at an index, common nouns denote sets of individuals, we can identify them only under certain guises - that is, we need individual concepts to pick out individuals. Aloni captures this in terms of conceptual covers over the domain of individuals. In order to capture the semantics of change, I deviate from her formulation by introducing temporality. I assume that the set of

\footnotetext{
${ }^{11}$ Nathan (2005) and Romero (2006) term the distinction 'relational' vs. 'non-relational'. Since they do not offer clear criteria for relationality, I stick to Barker's (1995) individuation of relational nouns as those that allow for of NP arguments (bodyguard of Arnold/*tree of Arnold). Then, PC vs. SC depends on how easily a 'role' reading is available instead of an 'occupant'-reading. That is, while both bodyguard and governor are relational, only governors are easily taken to fulfill a particular office each.

${ }^{12}$ Romero (2006), achieves an elegant solution in the Montagovian framework for examples with definite descriptions. Nevertheless, as she herself points out, it cannot be generalized to (other) quantificational determiners.

${ }^{13}$ I am indebted to Ede Zimmermann (p.c.) for drawing my attention to the potential relevance of this body of work to the topic under investigation.
} 
intervals $T$ comes with a partial order $\sqsubseteq$. The atomic parts (temporal instants) $T_{A}$ form the (left) domain of the ordering ${ }_{A}$ defined by $t^{\prime} \sqsubseteq_{A} t$ iff $t^{\prime} \sqsubseteq t$ and there is no $t^{\prime \prime}$ s.t. $t^{\prime \prime} \neq t^{\prime}$ and $t^{\prime \prime} \sqsubseteq t^{\prime}$.

(30) Given a set of indices (pairs of worlds $W$ and temporal instants $T_{A}$ ) and a universe of individuals $D$, a conceptual cover $\boldsymbol{C} C$ based on $\left(W \times T_{A}, D\right)$ is a set of functions $\left(W \times T_{A}\right) \rightarrow D$ such that:

$\left(\forall(w, t) \in W \times T_{A}\right)(\forall d \in D)(\exists ! c \in C C)[c(w, t)=d]$

A conceptual cover $\mathrm{CC}$ is thus a set of individual concepts that obeys two restrictions: (i) at each index, all individuals are picked out (existence), and (ii) at each index, each individual is picked out by only one individual concept (uniqueness).

Which cover is salient depends on the contextual perspective. Consider (31). Depending on the context of the conversation, on some occasions (31a) counts as a good answer (e.g. at a history exam), on others (31b) does (e.g. at a cocktail party where the interrogator might know perfectly well the name of the person she is looking for).

(31) Who is the president of Mali?

a. Amadou Toumani Touré.

at a history exam

b. Him! (pointing at someone)

at a cocktail reception

The contrast can be captured naturally if it is assumed that the partitioning induced by a question (cf. Groenendijk and Stokhof 1984) is sensitive to the salient conceptual cover. (31a) resolves the question if the naming cover (32a) is salient, and (31b) does if a rigid cover is (32b) ( $K$ is the set of proper names):
a. $\mathrm{NC}=\left\{\lambda(w, t) . \iota x\left[\exists t^{\prime}\left[x\right.\right.\right.$ is called $a$ in $w$ at $\left.\left.\left.t^{\prime}\right]\right] \mid a \in K\right\}$
naming cover
b. $\mathrm{RC}=\{\lambda(w, t) \cdot d \mid d \in D\}$
rigid cover, used in pointing

Under the assumption that individuals are accessed only with respect to a particular conceptual cover, quantification, too, can be treated as sensitive to conceptual covers. Even if, semantically, the restrictor denotes a set of individuals, the nuclear scope predicate can be applied to the individual concepts used to pick them out. I add the salient contextual cover $F$ as a parameter of interpretation. ${ }^{14}$

I assume that change is true of an individual concept $f$ in $w$ at an interval $t$ iff $t$ contains at least two points which are assigned different values by $f .{ }^{15}$

$$
\llbracket \text { change } \rrbracket(w, t)(f)=1 \text { iff } \exists t^{\prime}, t^{\prime} \sqsubseteq_{A} t: f\left(w, t^{\prime}\right) \neq f\left(w, t^{\prime \prime}\right) .
$$

$F[w, t]$ is pointwise application of a set of functions $F=\left\{f_{1}, \ldots, f_{n}\right\}$ to $(w, t)$ :

\footnotetext{
${ }^{14}$ This is a slight simplification we can take since we are only concerned with sentences that are interpreted w.r.t. one cover (at a time).

${ }^{15}$ Instead of the semantics in (9), rise is now also intepreted at an interval, e.g. as in (i):

(i) $\llbracket$ rise $\rrbracket(w, t)\left(f_{\langle s, e\rangle}\right)=1$ iff $f($ left $(t))<f($ right $(t))$, where left and right map a closed interval onto its left and right boundary respectively.
} 


$$
F[w, t]:=\left\{f_{i}(w, t) \mid f_{i} \in F\right\}
$$

In general, quantificational determiners are interpreted as in (35a) (MOST/EVERY/3 stand for the $\langle\langle\langle s, e\rangle, t\rangle,\langle\langle\langle s, e\rangle, t\rangle, t\rangle\rangle$ versions of the standard interpretations). That is, they quantify over the elements in that part of the conceptual cover that picks out the individuals constituting the extension of the restrictor predicate throughout the reference interval. The extension of common nouns may change in the course of the reference interval $t$, therefore, determiners force us to interpret them at each instant within such an interval. I call a subset of a conceptual cover $F$ that picks out exactly the extension of a predicate $P$ (at all atomic parts of an interval $t$ ) a $\boldsymbol{P}$-cover (for $t) .{ }^{16}$ Now, three, for example, comes out as in (35b).

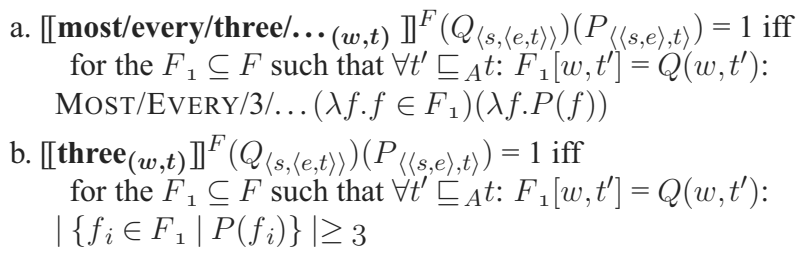

\subsection{Accounting for Nathan's Puzzle and Other Observations}

We are now ready to explain the contrast between set change and pointwise change as observed in (36) vs. (37):
a. Three bodyguards changed.
b. Three pictures on Jordan's wall have changed.

Three mayors changed.

As discussed in Section 3.4, nouns like bodyguard or picture on the wall give rise to set-change interpretations. I argue that this is the case because we tend to think of them as individuals simpliciter and thus pick them out with a temporally constant cover that is inapplicable to describe the relevant sets in the scenario of change. A (subset of a) conceptual cover CC is temporally constant iff it does not contain any individual concepts that for one and the same world assign different values at different temporal instants:

(38) If $C C$ is a cover, then $F \subseteq C C$ is temporally constant iff for all $f_{i} \in F$, $w \in W, t, t^{\prime} \sqsubseteq_{A} T: f_{i}(w, t)=f_{i}\left(w, t^{\prime}\right)$, else $F$ is temporally variable.

(36a) is interpreted as true at $(w, t)$ iff $F_{1}$, the part of the salient cover $F$ that describes the bodyguards throughout the reference interval $t$, contains at least three individual concepts that assign different values to at least two instants within $t$ :

$$
\begin{aligned}
& \llbracket \operatorname{three}_{(w, t)}\left(\lambda(w, t) . \text { bodyguards }_{(w, t)}\right) \text { change }_{(w, t)} \rrbracket^{F}=1 \text { iff } \\
& \quad \text { for the } F_{1} \subseteq F \text { such that }
\end{aligned}
$$

\footnotetext{
${ }^{16}$ Note that, for any $P$, a conceptual cover $\mathrm{F}$ will contain at most one $P$-cover. If it does not contain one, interpretation with respect to that particular $F$ fails.
} 


$$
\begin{array}{r}
\text { for all } t^{\prime} \sqsubseteq_{A} t: F_{1}\left[w, t^{\prime}\right]=\llbracket \text { bodyguard } \rrbracket^{F}\left(w, t^{\prime}\right): \\
\left|\left\{f_{i} \in F_{1} \mid \exists t^{\prime}, t^{\prime \prime} \sqsubseteq_{A} t: f_{i}\left(w, t^{\prime}\right) \neq f_{i}\left(w, t^{\prime \prime}\right)\right\}\right| \geq 3
\end{array}
$$

Assume that (36a) is interpreted in scenario like (40) where $t$ consists of two subintervals $t^{\prime}$ followed by $t^{\prime \prime}$, such that after $t^{\prime}$, John, Peter and Mary are exchanged against Simon, Susi and Sandro, while Sally remains as a bodyguard throughout both parts of $t$. Intuitively, the sentence should be true under these circumstances.

$$
\begin{aligned}
& \llbracket \text { bodyguard } \rrbracket^{F}\left(w, t^{\prime}\right)=\{\text { john, peter, mary, sally }\} \\
& \text { [bodyguard } \rrbracket^{F}\left(w, t^{\prime \prime}\right)=\{\text { simon, susi, sandro, sally }\}
\end{aligned}
$$

Now, crucially, bodyguards or pictures on a wall tend to be perceived as sets of individuals - we use either naming or pointing covers to individuate them. Both are temporally constant.Therefore, neither the naming cover $N C=\left\{\lambda(w, t) . \iota x\left[\exists t^{\prime}[x\right.\right.$ is called 'john' at $\left.\left.\left(w, t^{\prime}\right)\right]\right], \lambda(w, t) . \iota x\left[\exists t^{\prime}\left[x\right.\right.$ is called 'peter' at $\left.\left.\left.\left(w, t^{\prime}\right)\right]\right], \ldots\right\}$, nor the rigid cover used in pointing contains a subset $F_{1}$ that describes the bodyguards throughout $t$. Therefore, interpretation fails with respect to $F$. But since $F$ is just a matter of contextual salience, I assume that hearers try to envoke a more sensible cover. To capture this, I adopt the the Principle of Cooperative Indentification.

(41) Principle of Cooperative Identification: Consider a quantified sentence $p=$

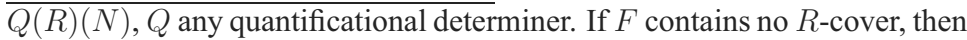
$\llbracket p \rrbracket^{F}=\llbracket p \rrbracket^{F^{\prime}}$, where $F^{\prime}$ is the next salient CC after $F$ s.t. $F^{\prime}$ contains an $R$-cover. If no CC salient in the context contains an $R$-cover, $\llbracket p \rrbracket^{F}=1$ iff $\llbracket \mathrm{p} \rrbracket \rrbracket^{F^{\prime}}=1$ for each arbitrary CC $F^{\prime}$ that contains an $R$-cover.

Considering all possibilities of arbitrarily aligning the individuals in the restrictor's denotation amounts to supervaluation over all ways to describe the set of bodyguards throughout the reference interval. Our case involves four individuals, the sentence thus has to be true w.r.t. 4! types of arbitrary conceptual covers (variation on non-bodyguard individuals is ignored as irrelevant). Since three of the bodyguards get exchanged, none of these 24 covers can contain more than one individual concept that does not change from $t^{\prime}$ to $t^{\prime \prime}$ (the only candidate is $\lambda(w, t) . \iota x\left[\exists t^{\prime}[x\right.$ is called 'sally' at $\left.\left.\left.\left(w, t^{\prime}\right)\right]\right]\right)$. But if fewer than three bodyguards are exchanged, some arbitrary alignments will contain less than three changing individual concepts and will falsify the sentence. Therefore, the sentence can only be true if the set changes by the indicated amount.

Let us now look at mayors. In contrast to bodyguards or pictures, they easily give rise to temporally variable conceptual covers. Often, we think of mayors not just as the individuals who happen to be mayor at a certain point in time, but in terms of their particular job. Therefore, change in the set can be irrelevant, as long as there is pointwise change with respect to the functions we have used to individuate the individuals in question. Mayors render salient either the naming cover $\mathrm{NC}$, or a job cover depending on cities. Both can serve to identify the individuals who are mayor at a particular point of time.

$$
\begin{aligned}
& \mathrm{NC}=\left\{\lambda(w, t) . \iota x\left[\exists t^{\prime}\left[x \text { is called 'klaus' at }\left(w, t^{\prime}\right)\right]\right], \lambda(w, t) . \iota x\left[\exists t^{\prime}[x \text { is called }\right.\right. \\
& \text { 'petra' at } \left.\left.\left.\left(w, t^{\prime}\right)\right]\right], \ldots\right\}
\end{aligned}
$$




$$
\mathrm{JC}=\left\{\lambda(w, t) . \iota u\left[\text { mayor-of-frankfurt }_{(w, t)}(u)\right], \lambda(w, t) . \iota u\left[\text { mayor-of-berlin }_{(w, t)}(u)\right], \ldots\right\}
$$

Consider (44) at $(w, t)$, where Klaus and Petra simply exchange their cities, without there being a change in the overall set of mayors.

$$
\begin{aligned}
& \llbracket \operatorname{two}_{(\boldsymbol{w}, t)}\left(\boldsymbol{\lambda}(\boldsymbol{w}, \boldsymbol{t}) \cdot \operatorname{mayors}_{(\boldsymbol{w}, t)}\right) \text { changed }_{(\boldsymbol{w}, t)} \rrbracket^{F}=1 \text { iff } \\
& \quad \text { for the } F_{1} \subseteq F \text { such that } \forall t^{\prime} \sqsubseteq A t: F_{1}\left[w, t^{\prime}\right]=\llbracket \operatorname{mayor} \rrbracket^{F}\left(w, t^{\prime}\right): \\
& \left|\left\{f_{i} \in F_{1} \mid \exists t^{\prime}, t^{\prime \prime} \sqsubseteq A t: f_{i}\left(w, t^{\prime}\right) \neq f_{i}\left(w, t^{\prime \prime}\right)\right\}\right| \geq 2
\end{aligned}
$$

Consider the table in (45). If the job cover is salient (that is, $F=J C$ ), the sentence comes out as true. Therefore, pointwise change is normally sufficient. But if for some reason the naming cover is more salient, the sentence in (44) comes out as false. And indeed, speakers tend to judge it as false in a scenario which forces interest in the bare individuals (e.g. which politicians should get invited to an annual $\mathrm{BBQ})$.

mayors at $(w, t)$, where $t$ consists of $t^{\prime}$ followed by $t^{\prime \prime}:$
\begin{tabular}{|l||ll||ll|}
\hline \multicolumn{1}{|c||}{} & \multicolumn{3}{c||}{ JC } & \multicolumn{2}{c|}{ NC } \\
& mayor Frankfurt & mayor Stuttgart $_{\text {F }}$ & Klaus & Petra \\
\hline$\left(w, t^{\prime}\right)$ & klaus & petra & petra & klaus \\
$\left(w, t^{\prime \prime}\right)$ & klaus & petra & klaus & petra \\
\hline
\end{tabular}

We are now able to account for common nouns with $\langle e, t\rangle$-denotations as restrictors of quantifiers in intensional argument positions, and also for Nathan's Puzzle. The solution is inherently pragmatic: Interpretation depends on the perspective taken on the individuals in the context. But is such a pragmatic solution strong enough to account for the temperature paradox? Consider first the premise of the fallacy where the intensional interpretation of the subject noun phrase has to come in (46).

(46) The temperature is rising.

To interpret this, we have to first saturate the free variable, and interpret temperature as temperature of a contextually salient location. The truth conditions with respect to the contextually salient cover $F$ are now computed as follows:

$$
\begin{aligned}
& \llbracket \text { the }_{(\boldsymbol{w}, t)}(\boldsymbol{\lambda}(\boldsymbol{w}, \boldsymbol{t}) \text {.temperature } \\
& (\boldsymbol{w}, t) \\
& \text { the } F_{1} \subseteq F \text { such that } \forall t^{\prime} \sqsubseteq A t: F_{1}\left[w, t^{\prime}\right]=\llbracket \text { rise }_{(\boldsymbol{w}, t)} \rrbracket^{g, F}(w, t)=1 \text { iff for } \\
& \left|F_{1}\right|=1 \text { and } \forall f_{i} \in F_{1}: f_{i} \in \llbracket \text { rise } \rrbracket^{g, F}(w, t) .
\end{aligned}
$$

But shouldn't this enable us to understand (48) as saying that the temperature of Frankfurt is rising, provided that temperatures are picked out as for (47), and the actual temperature of Frankfurt is known to be 90 ?

Ninety is rising.

At first glance, this may look like a knock-down argument against the pragmatic solution, but, at second glance, it even provides a strong argument in its favour. Crucially, (48) does not contain a determiner, and the solution I am advocating relies on a determiner to introduce sensitivity to covers. Compare (49): ${ }^{17}$

\footnotetext{
${ }^{17}$ The facts were first pointed out to me by Felix Schumann (p.c.) for German. I am also indebted to Samson Tikitu de Jager for bringing to my attention that the same facts hold in English.
} 
(49) The temperature in Ede's office is already 32 degrees and I think

a. ...*(the) 32 degrees will certainly increase.

b. ...*(that) 32 degrees is going up by mid-afternoon.

If a particular value has been introduced in relation to a temperature function, reference to that temperature function is possible by a definite description that uses the name of the value as the descriptive part. That is, 32 degrees refers to the individual directly, but a cover containing $\lambda(w, t)$.the-temp.-in-Ede's-office $(w, t)$ is used when evaluating the 32 degrees. Since $\lambda(w, t)$.the-temp.-in-Ede's-office $(w, t)$ need not be temporally constant, (49) is felicitous and can be true.

While (48) and (49) corroborate the analysis that determiners are responsible for bringing in individual concepts, Maribel Romero (p.c.) points out (50) as problematic: As I translate temperature as type $\langle s,\langle e, t\rangle\rangle,(50)$ seems to pair rise with a variable of type $e$ inside the relative clause.

(50) Most temperatures that haven't risen in a long time are rising now.

Under the traditional head-external construal of relative clauses, (most) temperatures is generated outside the relative clause. The relative clause contains an operator trace of type $e$ and as a whole, expresses a $\langle e, t\rangle$ predicate fit to restrict the external head temperature. But Safir (1999) argues convincingly that reconstruction (and, hence, a copy inside of the relative clause) is necessary to explain the parallel behaviour of external heads and relative clause operator phrases with respect to reconstruction effects. ${ }^{18}$ Thus, a head-external analysis along the lines of (51b) is excluded. The LFs claimed to be available instead are interpretable under the typing I propose. The relevant copy of the external head temperatures is the one that appears as the complement of the relative clause predicate (cf. 51a). Even for $\langle e, t\rangle$-predicates, interpretation would fail. To render the structure interpretable, Fox (2002) proposes trace conversion as an operation at LF. The process inserts a definite determiner in front of the relative clause internal ('reconstructed') copy of temperatures. Its index is interpreted as identity with the variable abstracted over, which by Predicate Modification restricts this lower copy of temperature.
a. most [ temperatures [ that are rising ]]
b. most [ temperatures $\lambda$ x.rise $(w, t)(\mathrm{x})]$
c. most [ temperatures [ $\lambda \mathrm{x}[$ the $\lambda \mathrm{y}[$ temperature $(\mathrm{y}) \& \mathrm{x}=\mathrm{y}]]]]$

Translation and interpretation for (51c) are given in (52). Note that to capture relative clauses, we have to allow the index of the predicate in the relative clause to be interpreted with respect to the matrix clause. Under the only sensible interpretation, it is not bound by the index binder arising in quantifiction. ${ }^{19}$

\footnotetext{
${ }^{18}$ Roughly, quantifiers in argument but not in adjunct position of either external heads and operator phrases of relative clauses give rise to secondary crossover effects.

${ }^{19}$ Otherwise, individual concepts would have to rise at a temporal instant, which is inconsistent with the semantics adopted for rise. Technically, this requires a refined treatment of world and time variables as offered e.g. by Percus (2000). He also provides a natural starting point for exploring what the constraints are in these cases.
} 


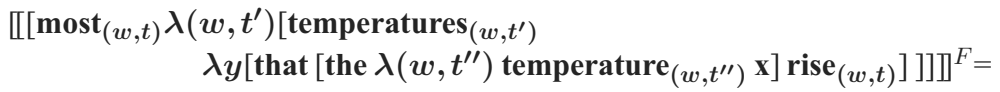

$\lambda Q$.for the $F_{1} \subseteq F: \forall t^{\prime} \sqsubseteq A t: F_{1}\left[w, t^{\prime}\right]=\lambda y$. [temperature $\rrbracket^{F}\left(w, t^{\prime}\right)(y)$ and the $F_{2} \subseteq F: \forall t^{\prime \prime} \sqsubseteq A t^{\prime}: F_{2}\left[w, t^{\prime \prime}\right]=\lambda x$. [temperature $]^{F}\left(w, t^{\prime \prime}\right)(x)$ and $x=y$ :

$\left|F_{2}\right|=1$ and $\forall f \in F_{2}: \llbracket$ rise $\rrbracket^{F}(w, t)(f): \operatorname{MosT}\left(\lambda f . f \in F_{1}\right)(\lambda f . Q(f))$.

In (52), we quantify over that cover part $F_{1}$ that at all instants within $t$ picks out those temperatures $x$ such that the individual concept that picks out $x$ at that instant is rising at the reference interval $(w, t)$. This seems to be the correct reading.

The pragmatic analysis also squares well with the amount of context dependence observed in whether a noun receives a set-change or a point-wise change interpretation. Although pictures on Jordan's wall has a strong tendency to be interpreted as inducing set-change, some speakers argue that they could also think of pictures in terms of their positions in Jordan's room (the picture closest to the window, the picture above the bed, the picture just left of the door,...), and in that case, get an interpretation of pointwise change. The same holds for bodyguards: if each of them has a fixed responsibility (e.g. one guards the back door, one takes care of the car,...), then all of a sudden pointwise change becomes available. This volatility is entirely expected under the pragmatic account, but hard to capture if individual concepts come from the lexicon. Nevertheless, it is far from trivial to predict what conceptual cover(s) is/are salient in a given situation. Aloni (2005) constitutes the most elaborate attempt to provide a stringent formal pragmatic account to determine which conceptual cover is actually used in a particular context. The approach is couched in bi-directional OT and makes quite good predictions. Yet, it is acknowledged that it does not capture all scenarios considered.

\section{A Problem for Temperatures as Individuals}

For the examples considered so far, the pragmatic solution seems to make the right predictions. Let us now consider an ambiguity that, while providing further corroboration at first glance, turns out to be highly problematic.

(53) The lowest temperature is rising.

On one reading (call it the city reading), the sentence is true of a scenario where we are interested in the temperatures of three cities, e.g. Frankfurt, New York, and Amsterdam. Then, (53) is true if the city that happens to have the lowest temperature at the moment of evaluation is such that its temperature is rising (consequently, it might soon cease to be the coldest place). On the other reading (call it the ranking reading), the sentence is true of a scenario where we are recording the temperatures of maybe different cities every day and realize that from day to day, the lower boundary of our measurements is going up (providing evidence for 
theories of global warming). ${ }^{20}$ The ambiguity is highly reminiscent of an example Heim (1979) discusses for concealed questions. (54) is ambiguous between a plain reading where John and Fred (maybe without being aware of each other's existence even) are able to answer the same price-question, and a higher order reading, where John is able to indicate which price-question Fred is able to answer (without maybe John himself being able to answer that price-question).

John knows the price Fred knows.

Heim observes that the existence of the higher order reading constitutes a serious problem for a pragmatic solution to concealed questions in terms of de re-attribution of a property to an individual (cf. Heim 1979, Frana 2006; Schwager 2007 for a recent attempt to remedy that problem). In contrast, Romero (2005) obtains an elegant solution for (54) by using both individual concepts coming from the lexicon and individual concepts resulting as intensions of definite descriptions. Independently of the problem the pragmatic theory of concealed questions faces with the ambiguity in (54), at first glance, the pragmatic access to individual concept seems to work perfectly for the ambiguity in (53). We expect to obtain the city reading, if the salient conceptual cover looks like (55a), and the ranking reading if the salient cover contains the denotation of the subject noun phrase and looks like (55b).

$$
\begin{aligned}
& \text { a. city-cover }=\{\lambda(w, t) \text {.the-temp.-of-Frankfurt }(w, t), \\
& \left.\quad \lambda(w, t) \text {.the-temp.-of-Amsterdam }(w, t), \lambda(w, t) \text {.the-temp.-of- } N Y_{(w, t)}\right\} \\
& \text { b. ranking-cover }=\{\lambda(w, t) \text {.the-highest-temp. }(w, t), \\
& \left.\quad \lambda(w, t) . \text { the- } 2^{\text {nd }} \text {-highest-temp. }(w, t), \lambda(w, t) \text {.the-lowest-temp. }(w, t)\right\}
\end{aligned}
$$

But now, assume that, at some index, two cities happen to have the same temperature index. Consider (56):

(56) Three temperatures are rising.

According to my pragmatic solution, we first collect all abstract degree individuals that are temperatures (of an object in a contextually given set) for the index of evaluation $(w, t)$. Then, we have to establish a suitable conceptual cover for this set of individuals and check if three individual concepts within that cover are rising at $(w, t)$. Therefore, if at $(w, t)$, the temperature of Frankfurt is the same as the temperature of New York (e.g. 20C), a suitable conceptual cover should contain only one individual concept to pick out these 20C. And, due to uniqueness, (55a) does not consitute a conceptual cover of these abstract degree individuals. But this runs counter to our intuitions: we want to simply count cities with rising temperatures and ignore whether two of them are at the exactly same level at the point of interest. In contrast, the ranking reading is indeed blind to the fact how many cities instantiate a certain value. (53) is still felicitous under the ranking reading if the lowest temperature measured is displayed by two cities. If individuation by city is the only cover available, (53) results in a presupposition failure.

\footnotetext{
${ }^{20}$ Note that the two readings are truly independent: the ranking reading entails the city reading only if we are measuring the same cities' temperatures every day.
} 
Currently, the only solution I can think of is to reconsider what temperatures actually are. We make the right predictions for the city reading if temperature individuals are concrete events (like tropes in the sense of e.g. Moltmann 2004). Nevertheless, for the ranking reading, we have to retain temperature individuals as abstract values. At first glance it might seem implausible that there are now two levels at which pragmatics influences quantification: on the one hand, it is sensitive to how we pick out the individuals in question, but on the other hand, it is also sensitive to what are individuals in the first place, and, moreover, these two issues are not independent. Nevertheless, the second kind of dependence is found in purely extensional contexts, too. Consider counting ambiguities as recently discussed in detail by Asher (2007).

a. Julie mastered every book in the library.

b. Julie carried off every book in the library.

While (57a) tends to count types of books, (57b) tends to count individual copies. In this case it is the predicate that determines the granularity of counting. For (53), both granularities seem to be fine, but the granularity seems to go hand in hand with what constitute possible covers. At this point, I can only leave the issue for future research. But I am optimistic that a better understanding of counting puzzles like (57) and the salience of particular conceptual covers will go together to enlighten these problems for the pragmatic solution.

\section{Conclusions}

I have argued that function readings for nouns like temperature or mayor depend on how we individuate individuals that are temperatures and mayors at the indices of interest. This provides a solution to classical problems like the temperature-paradox and, without meaning postulates, avoids multiplicity of such functions across indices. Moreover, it explains why change is normally interpreted pointwise with nouns like mayor, but setwise with bodyguards (Nathan's puzzle). The dependence of the effect on the utterance context is captured naturally. Nevertheless, the interdependence of particular covers and what count as individuals of a certain type, is still ill understood, as well as the question what determines what conceptual cover(s) are salient.

\section{References}

Aloni, Maria: 2000, Quantification under Conceptual Covers. ILLC, Amsterdam.

Aloni, Maria: 2005, 'A formal treatment of the pragmatics of questions and attitudes', Linguistics and Philosophy 28, 505-539.

Asher, Nicholas: 2007, 'A Web of Words: Lexical Meaning in Context'. Draft (book), CNRS/UT Austin, June 2, 2007. 
Barker, Chris: 1995, Possessive Descriptions. CSLI Publications, Stanford.

Dowty, David R, Robert E Wall, and Stanley Peters: 1981, Introduction to Montague Semantics. Reidel, Dordrecht.

Fox, Danny: 2002, 'Antecedent Contained Deletion and the Copy Theory of Movement', Linguistic Inquiry 33, 63-96.

Frana, Ilaria: 2006, 'Wondering about Concealed Questions'. Talk at SALT 16, University of Tokyo, March 22-24, 2006.

Groenendijk, Jeroen and Martin Stokhof: 1984, Studies on the Semantics of Questions and the Pragmatics of Answers, Doctoral Dissertation, Amsterdam.

Heim, Irene: 1979, 'Concealed Questions', in R. Bäuerle, U. Egli, and A. von Stechow (eds.), Semantics from Different Points of View, 51-60. Springer, Berlin.

Lasersohn, Peter: 2005, 'The Temperature Paradox as Evidence for a Presuppositional Analysis of Definite Descriptions', Linguistic Inquiry 36, 127-144.

Löbner, Sebastian: 1979, Intensionale Verben und Funktionalbegriffe. Narr, Tübingen.

Löbner, Sebastian: 1981, 'Intensional verbs and functional concepts: more on the 'rising temperature' problem', Linguistics Inquiry 12, 471-477.

Löbner, Sebastian: 1985, 'Definites', Journal of Semantics 4, 279-326.

Moltmann, Friederike: 2004, 'Properties and kinds of tropes: New linguistic facts and old philosophical insights', Mind 113, 1-41.

Montague, Richard: 1974, 'The Proper Treatment of Quantification in English', in Formal Philosophy. Selected Papers of Richard Montague., herausgegeben und mit einer einführung von R. H. Thomason. edn, 247-270. Yale University Press, New Haven/London.

Nathan, Lance: 2005, 'On the interpretation of concealed questions'. Doctoral Dissertation (draft), MIT.

Partee, Barbara: 1989, 'Binding Implicit Variables in Quantified Contexts', Papers of the Chicago Linguistics Society 25, 342-365.

Percus, Orin: 2000, 'Constraints on some other variables in syntax', Natural Language Semantics 8, 173-229.

Romero, Maribel: 2005, 'Concealed Questions', Linguistics and Philosophy 28, 687-737.

Romero, Maribel: 2006, 'Some Paradoxes About Individual Concepts'. Invited talk at Sinn und Bedeutung, September 21-23, 2006, Barcelona.

Safir, Ken: 1999, 'Vehicle Change and Reconstruction in A-Chains', Linguistic Inquiry 30, 587-620.

Schwager, Magdalena: 2006, 'The topic has changed: On functional concepts'. Talk at Semantiknetzwerktreffen VI, Barcelona, September 19-20.

Schwager, Magdalena: 2007, 'Keeping Prices Low'. Talk to be delivered at Sinn und Bedeutung XII, Oslo, September 20-22.

Zimmermann, Thomas Ede: 1999, 'Meaning Postulates and the Model-Theoretic Approach to Natural Language Semantics', Linguistics and Philosophy 22, 529-561. 